\title{
Vision Controlled Robotic System
}

\author{
Geetha Ramadas, Sukhi Y
}

\begin{abstract}
The field of robotics is changing the world in which we live today and the future generations will be largely served by various robotic services which will be highly efficient and dynamic. Offering affordable and efficient robotic services to the society is the need of the hour and this project is highly inclined towards this theme. The latest touch screen technology and the use of mechanical components like joysticks can be replaced by Gesture Controlled Robotics. The product is aimed at improving the conditions of motor neuron disabled persons using OpenCV libraries of Processing 3 software and Arduino Uno (Atmega328 microcontroller). The vision of the disabled person is sensed using a Glass Eye Tracker and every 25th frame of the picture (here it is the pupil) is sent to the Processing3 software. This software processes the image based on the algorithm. The ports of Processing 3 software and Arduino are serially communicated. Now the Arduino performs the output part with the help of L293D motor driver and 24V DC motor.
\end{abstract}

Keywords: Image processing, formatting, style, styling, insert.

\section{INTRODUCTION}

There are many applications of Image Processing System in the medical field. Electric motor is used to drive a motorized wheelchair, or electric powered wheel chair (EPW). Electric powered wheel chairs are used by those who are not able to propel a manual wheelchair. They need electric wheel chair for movement for long distances which may produce fatigue in a manual wheelchair. The motorized operation is more manually controlled in this world of automation. This has restricted the fullest potential of such motorized operations.

\section{METHODS}

\section{A. Existing System}

A motorized wheelchair is a wheel chair using electric motor rather than manual power. Motorized wheelchairs are used for a long distance movement which may produce fatigue in a manual wheelchair. The motorized operation is more manually controlled in this world of automation. This has restricted the fullest potential of such motorized operations.

\section{B. Proposed System}

The proposed system various techniques involved in the image processing and its application in the wheel chair using motor controller system. This system consolidate various techniques involved in image processing and provide an efficient data. The processed data is then fed to the ATmega328 processor for further signal classification. This serial communication occurs at 9600 baud rate. Then the output of the serial communication is applied to L293D motor driver which converts digital signals to analog signals(DAC) for the speed and rotation control of the motor

\section{IMAGE PROCESSING METHOD}

Image processing is a method of signal processing to process images. The images are converted into digital signals in image processing and some more operations are done on it to enhance the image or to get some information from it.

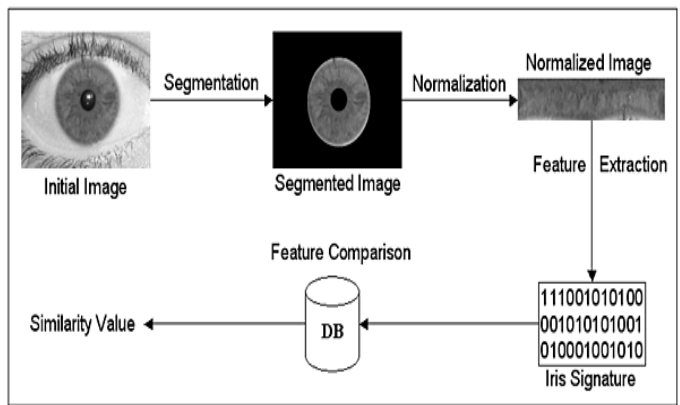

Fig. 1. Image Processing Method

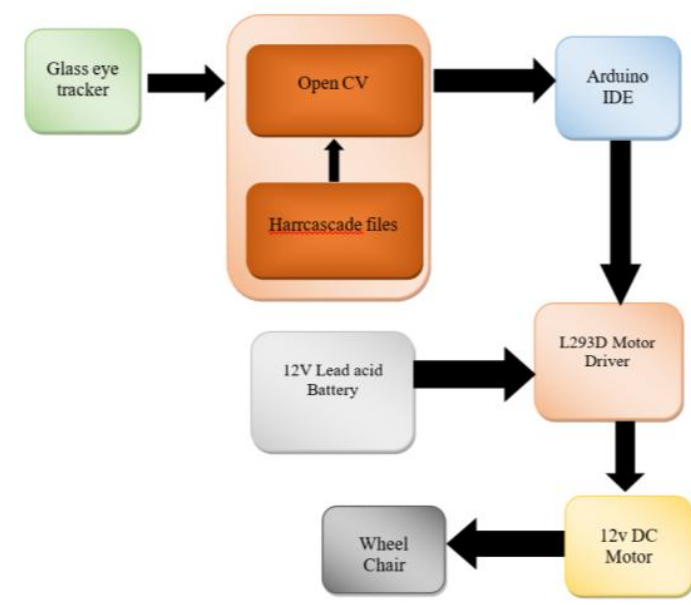

Fig. 2 Block Diagram of Image Processing

* Correspondence Author

Geetha Ramadas, Department of Electrical and Electronics Engineering, Kavaraipettai, Gummidipoondi Taluk, Thiruvallur District, Tamilnadu, India. Email: grs.eee@ rmkec.ac.in

Sukhi Y, Department of Electrical and Electronics Engineering, Kavaraipettai, Gummidipoondi Taluk, Thiruvallur District, Tamilnadu, India. Email: ysi.eee@rmkec.ac.in 


\section{Vision Controlled Robotic System}

Fig 1 shows the circuit involved in the processing. In this process the input is an image and output is a characteristic of the image or an image. In the image pprocessing system, signals are treated as images of two dimensional pictures to apply the previously available signal processing methods on it. Image processing is growing fast in the present technologies and is applied in various aspects of business. Image Processing is one of the important area of research in computer field and also in the engineering fields.

\section{DIFFERENT PHASES INVOLVED}

\section{A. Image Acquisition}

The general aim of Image Acquisition is to transform an optical image (Real World Data) into numerical data in a sequential way which can processed using the computer later. The image is captured by the camera and is converted into manageable entity before any video or image processing is done. Fig. 2 shows the process involved in the proposed system. Image Acquisition process totally depends on the hardware system which may have a sensor that is again a hardware device. Light signals are converted into electrical charges using sensors. The sensor inside a camera measures the reflected energy by the scene being imaged. Charge coupled device is used in image sensor. [1]. Complementary metal oxide is also used in cameras.

\section{B. Image Enhancement}

Image processing involves image enhancement which has some challenging works. In the vision applications, image enhancement plays a vital role. In this process of image enhancement of images the output image gives more specific information than the input image. The qualities of images are improved using digital image enhancement techniques. There are different techniques are available and the selection of appropriate technique is very important. The analysis of various techniques involved in the image enhancement is done.

\section{Image Restoration}

In image restoration, the original image is recovered from the corrupted images. It is widely used in optical communication and signal processing. This is done for compensation of images. In case of blur due to motion, actual blur is calculated using functions and the blur can be removed to obtain the original image.

When the images are damaged by external noise image compensation gives the best method for the degradation. The image reconstruction is done in the image restoration to get the uncorrupted image from external noise. When image processing is done for blurred images, blind deconvolution technique allows the recovery of the original image using spread function. The blurred image is represented using a sharp image with a constant as below

$$
\mathrm{y}=\mathrm{k} \Theta \mathrm{x}
$$

where $\mathrm{x}$ is a visually plausible sharp image, and $\mathrm{k}$ is a nonnegative blur kernel.

\section{Color Image Processing}

Color image processing is done to display image information for human perception. It is also fundamental step of digital image processing. It gives the applications of image processing in which extraction of the features from the image data, from which description, understanding and information can be obtained by machine. This system allows user to take hard copy of an image using printer and other output devices such as scanner etc., and also store the screen image into the disk file using file format (.jpg, .gif, .png etc.). It relates the image properties such as alteration and analysis of pictorial information. Nowadays image processing is used in our daily life. Interaction of human brain with the human eye is one of the most image processing systems. The main objective is to develop and implement color image processing which is necessary to operate upon images and visually enhance the images.

\section{E. Compression}

In image compression, encoding of image file is done to occupy the less space than the actual file. In this compression technique size of the image file is done without affecting the quality of the image. Compression of photographs affect the may affect the image quality. This helps to store and transfer image in an economical way. Neural network is a structure similar to the biological network of nerve cell within the brain. Data compression using bit rate reduction is done using encoding the information. This can be lossless or lossy. In case of lossless compression, bits are reduced after the identification and are eliminated. There are many techniques like cryptography, vector division, encoding. These techniques have its own advantages and disadvantages. In these techniques some amount of bits are cut for presenting the image. This is done for reducing the storage space and transmission with good quality. Patterns and symbols for large capacity pattern are used in compression methods.

\section{F. Segmentation}

Segmentation for movement condition in the image is done using the difference in the images. Spilit approach is used for segmentation for four-layer process. Watershed algorithm is one of the method to split the image in the first layer. This is used to separate objects using pixels. In the co-evolutionary process of the second layer merging of primary regions is done. This algorithm does not use function to find out the solution. It creates entity to make solutions.In order to connect residual regions meta-heuristic process is done in the third layer. Evolutionary algorithm is used in the fourth layer. It is a generic based algorithm for optimization. It uses the biological progress.

\section{WORKING}

It is proposed to change the locomotion of a typical Wheelchair into a completely vision controlled unit. Each responds to a different Cascade Classifier Trainer. The proposed method deals with eye ball movement. Recognition human faces using digital images are implemented for detection of face using computer. In this the size and place of objects 
in an image for a class is identified. The process is based on Viola-Jones Algorithm which processes 25th frame of the capture. A Glass eye tracker (1280X720 @25fps) is used for capturing the image ( i.e, the pupil). Once the Processing3 detects changes in current position with respect to the previous position of the pupil, the information is serially communicated to Arduino at a baud rate of $9600 \mathrm{symbol} / \mathrm{sec}$. On receiving this information the microcontroller sets the particular pin to high and thus the motor driver comes into action. In the IC L293D, there are two $\mathrm{H}$ bridge circuits which is used to rotate two motors separately We have used 24VDC, 160RPM, 10Nm electric motor. According to the instruction given the microcontroller and the motor driver will accordingly act, thus the robot moves in the direction where eye ball moves. The software is developed in such a way that when the user blinks twice the robot starts moving forward. The rate of blink mentioned here is faster than the normal blinking rate. When the eyeball moves towards left, the robot starts moving to the left. Similarly when the eyeball moves to the right, the robot moves to the right. When it moves upward, the robot moves backward and when the eye blinks thrice the robot stops. Fig. 3 describes the experimentation procedure of image processing.

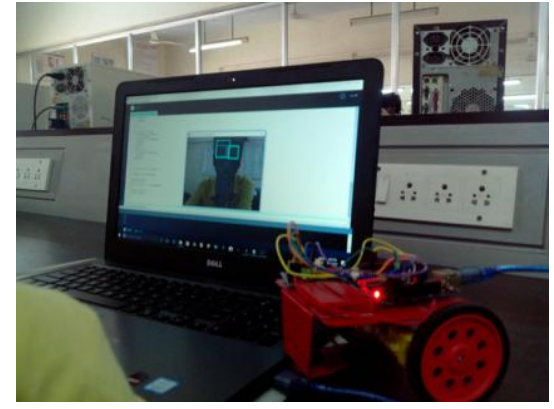

Fig. 3 Image processing experimentation

In this method we get much higher accuracy than using manual operation of motor system. This is due to the fact that we use computer vision thoroughly This gives better accuracy. Cost estimated is relatively lower than other automated wheel chair system. This results in lower cost. The major problem involved in this vision control is the battery backup facility. This requires battery backup.

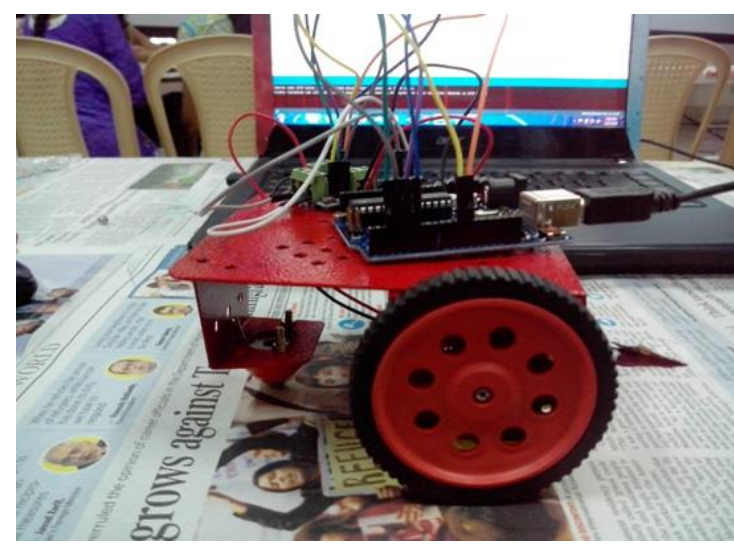

Fig. 5 Prototype of vision controlled robot
Fig. 5 shows the image processing output of the vision controlled robot. In this step, the Haarcascade files from processing library detect the feature required, that is the eye This process entirely focuses on the different phases involved in image processing and provides the necessary output to the motor control.

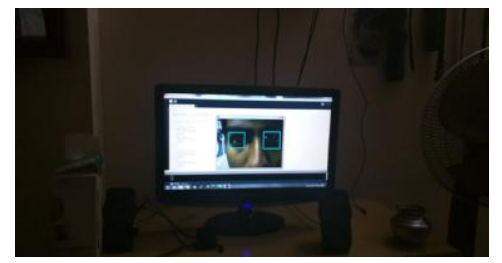

Fig. 4 Image Processing output

\section{CONCLUSION}

In this paper the method adopted for the proposed method is discussed. The prototype is developed for the process which we have done. This prototype emulates the basic movement which is performed by wheelchair. Here the Arduino IDE helps in analyzing the output from Processing3 Software and provides necessary control to the motor driver L293D.

\section{REFERENCES}

1. Maneesh Ayi1, Ajay Kamal Ganti, Maheswari Adimulam "Face Tracking and Recognition Using MATLAB and Arduino" January 2011

2. Rohan Chadha, Jai Prakash "Permanent Magnet DC Motor Control Using Image Processing" October 2011.

3. Ivan Matveev, Konstantin Gankin "Iris segmentation system based on approximate feature detection with subsequent refinements, 11 July 2017

4. Gulfishan Firdose Ahmed, Raju Barskar "A Study on Different Image Retrieval Techniques in Image Processing” December 2017.

5. Aniwat Juhong "Smart Eye-Tracking System", January 2018.

6. Sangeet Saha, Chandrajit pal, Rourab paul, Satyabrata Maity, Suman Sau "A brief experience on journey through hardware developments for image processing and it's applications on Cryptography" March 2018.

\section{AUTHORS PROFILE}

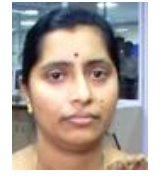

Geetha Ramadas received her B.Tech. Degree in Electrical Engineering from N S S College of Engineering, Palakkad / Calicut University in 1986. She obtained M.E in High Voltage Engineering from College of Engineering, Guindy, Anna University in 2002. She obtained $\mathrm{PhD}$ in Electrical Engineering from College of Engineering, Guindy, Anna University in 2002. Presently she is working as an Professor \& Head, Department of Electrical and Electronics Engineering. R.M.K Engineering College. Chennai. Tamilnadu, India.

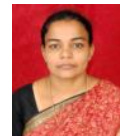

Sukhi Y received her B.E. Degree in Electrical and Electronics Engineering from Government College of Engineering / Madurai Kamaraj University in 1993. She obtained M.E in Mechatronics from Madras Institute of Technology, Anna University, Crompet in 2000. She obtained $\mathrm{PhD}$ in Electrical Engineering from Sathyabama University, Chennai in 2010. Presently she is working as an Professor, Department of Electrical and Electronics Engineering. R.M.K Engineering College. Chennai. Tamilnadu, India.. 